\title{
Megalencephaly and Macrocephaly
}

\author{
Kellen D. Winden, MD, $\mathrm{PhD}^{1}$ Christopher J. Yuskaitis, MD, $\mathrm{PhD}^{1}$ Annapurna Poduri, MD, MPH ${ }^{2}$ \\ ${ }^{1}$ Department of Neurology, Boston Children's Hospital, Boston, \\ Massachusetts \\ 2 Epilepsy Genetics Program, Division of Epilepsy and Clinical \\ Electrophysiology, Department of Neurology, Boston Children's \\ Hospital, Boston, Massachusetts

\begin{abstract}
Address for correspondence Annapurna Poduri, Epilepsy Genetics Program, Division of Epilepsy and Clinical Electrophysiology, Department of Neurology, Fegan 9, Boston Children's Hospital, 300 Longwood Avenue, Boston, MA 02115 (e-mail: Annapurna.Poduri@childrens.harvard.edu).
\end{abstract}

Semin Neurol 2015;35:277-287.

\begin{abstract}
Keywords

- megalencephaly

- hemimegalencephaly

- macrocephaly

- somatic mutation

Megalencephaly is a developmental disorder characterized by brain overgrowth secondary to increased size and/or numbers of neurons and glia. These disorders can be divided into metabolic and developmental categories based on their molecular etiologies. Metabolic megalencephalies are mostly caused by genetic defects in cellular metabolism, whereas developmental megalencephalies have recently been shown to be caused by alterations in signaling pathways that regulate neuronal replication, growth, and migration. These disorders often lead to epilepsy, developmental disabilities, and behavioral problems; specific disorders have associations with overgrowth or abnormalities in other tissues. The molecular underpinnings of many of these disorders are now understood, providing insight into how dysregulation of critical pathways leads to disease. The advances in molecular understanding are leading to improved diagnosis of these conditions, as well as providing new avenues for therapeutic interventions.
\end{abstract}

Megalencephaly is defined as a condition in which the size or weight of the brain is greater than two standard deviations above the age-related mean. ${ }^{1}$ This is in contrast to macrocephaly, which is defined based on increased orbitofrontal head circumference for age. The head circumference can be influenced by many factors other than the size of the brain, including skull size, subdural fluid collections, and ventricular size. Therefore, megalencephaly is a more specific term related to dysfunction in neurons or glia causing an abnormal size or number of these cells. Another major distinction is that macrocephaly can be isolated and benign, such as in benign familial macrocephaly, whereas megalencephaly is more often syndromic and unlikely to be benign. ${ }^{1}$ In particular, megalencephaly is often associated with developmental disabilities and epilepsy, which can be medically refractory. In clinical practice, the distinction between megalencephaly and macrocephaly relies on neuroimaging studies to identify enlarged cerebral structures or associated anomalies (-Fig. 1). However, distinguishing these conditions is important clinically in terms of diagnosis, further testing, and overall prognosis for the patient and family. ${ }^{2}$

Issue Theme Etiology of Epilepsy; Guest Editors: Philip Smith, MD, FRCP,

FAcadMEd, and Rhys Thomas, BSc, MRCP, MSc, PhD

Classically, megalencephaly has been divided into two categories: metabolic and anatomic. ${ }^{1}$ The metabolic megalencephalies encompass multiple disorders featuring accumulation of abnormal metabolites, whereas anatomic megalencephaly was described as increased size and/or number of cells without an identifiable metabolic abnormality. Both forms of megalencephaly have underlying genetic etiologies, and identification of causative single gene defects in these disorders has largely supported the original classification system. These studies have clarified underlying mechanisms, as well as provided new concepts for further study. Metabolic defects leading to megalencephaly are often caused by germline mutations and generally cause diffuse abnormalities in the brain, although there are examples of asymmetric involvement.

We will refer to disorders previously characterized as anatomic megalencephaly as developmental megalencephaly because the current identified genetic causes involve signaling mediators that regulate cell growth, migration, and replication during development. ${ }^{3,4}$ Development of the cerebral cortex is characterized by massive proliferation,

Copyright (C) 2015 by Thieme Medical Publishers, Inc., 333 Seventh Avenue, New York, NY 10001, USA. Tel: +1(212) 584-4662.
DOI http://dx.doi.org/ $10.1055 / \mathrm{s}-0035-1552622$. ISSN $0271-8235$. 


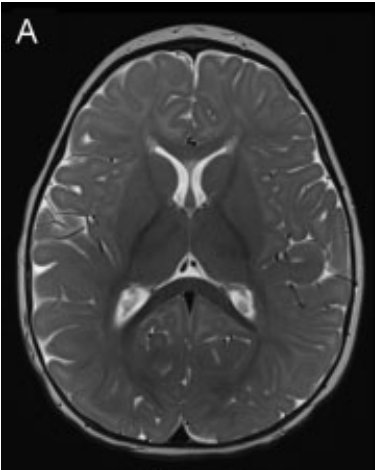

Normal

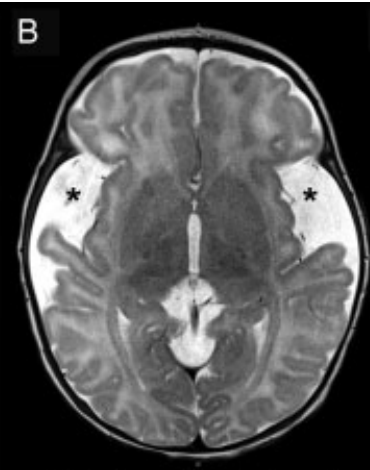

Glutaric aciduria, type I

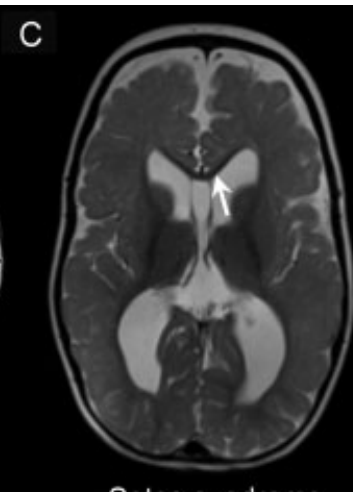

Sotos syndrome

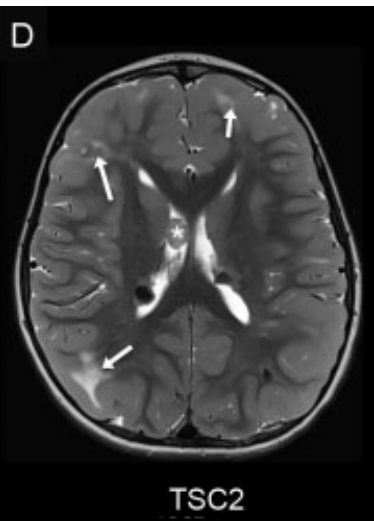

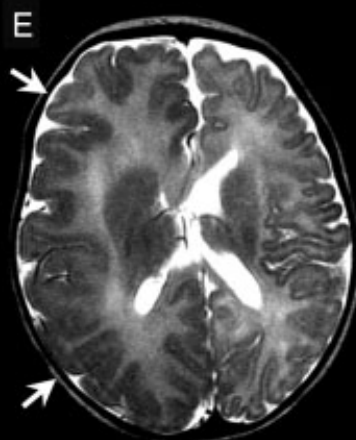

Hemimegalencephaly

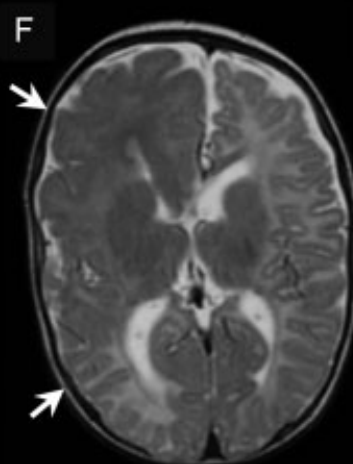

Hemimegalencephaly

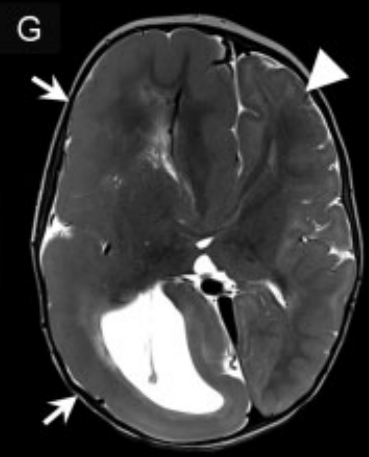

MCAP

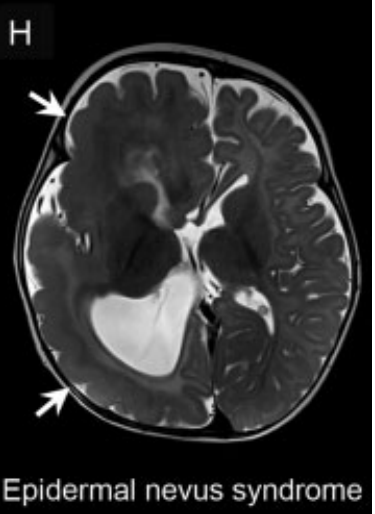

Fig. 1 Neuroimaging of metabolic and developmental megalencephaly. Representative axial T2-weighted magnetic resonance images: (A) A neurologically normal 1-year-old individual with symmetric hemispheres and normal myelination pattern for age. (B) An individual with glutaric academia, type I with typical features including enlarged extra-axial spaces and hypoplasia of the temporal lobes (asterisks), and delayed myelination pattern. (C) An individual with Sotos syndrome showing dolichocephaly and thinning of the corpus callosum (arrow). (D) An individual with tuberous sclerosis complex as a result of a TSC2 mutation. Note typical features including the multiple cortical tubers in the bilateral frontal and right parietal lobes (arrows), and the subependymal giant cell astrocytoma (SEGA) (asterisk). (E) Hemimegalencephaly of unknown etiology, with symmetric enlargement of one hemisphere of the brain (arrows). (F) AKT3 mutation resulting in asymmetric hemimegalencephaly, with arrows indicating enlargement of one hemisphere of the brain. (G) An individual with megalencephaly-capillary malformation-polymicrogyria syndrome (MCAP) showing hemimegalencephaly (arrows) and an abnormal gyration pattern on the contralateral side as well (arrowhead). (H) An individual with epidermal nevus syndrome that demonstrates asymmetric enlargement of one hemisphere (arrows).

differentiation, migration, and ultimately appropriate organization of neurons and glia. Neural progenitor proliferation, differentiation, and migration are controlled by multiple intrinsic and extrinsic signaling pathways that continually overlap during development. Classification of disorders is based on the earliest abnormal step, with the caveat that cells with proliferative defects often do not migrate or organize properly. ${ }^{5}$ Focal involvement is more common in the developmental megalencephalies; recently, somatic mosaicism has also been shown to play an important role in these diseases. $^{6,7}$

\section{Metabolic Megalencephaly}

Although metabolic diseases can be associated with both micro- and macrocephaly, there are several that are clearly associated with megalencephaly (-Table 1). However, not all patients with these disorders have megalencephaly, as there is often subsequent brain atrophy secondary to cell death. $^{3}$ Despite the fact that these diseases arise from critical metabolic pathways, there is often a limited range of cells that are affected. For example, leukodystrophies or disorders of the white matter, such as Canavan disease, Alexander disease, and megalencephalic leukoencephalopathy with subcortical cysts preferentially affect oligodendrocytes and astrocytes.

Canavan disease is a disorder of glial degeneration caused by mutations in the gene ASPA that encodes the enzyme aspartoacylase. ${ }^{8}$ Affected infants often appear normal until age 3 to 6 months, when they present with hypotonia and then rapidly progress to have limb spasticity with continued axial hypotonia and seizures. ${ }^{9}$ The aspartoacylase enzyme catalyzes the hydrolysis of N-acetylaspartic acid (NAA) to acetate. $\mathrm{N}$-acetylaspartic acid is synthesized by and transported into the extracellular region by neurons, where it is internalized by oligodendrocytes and metabolized. ${ }^{10}$ Loss of function of aspartoacylase leads to accumulation of NAA in oligodendrocytes; elevated NAA excretion in urine is sufficient to diagnose the condition. ${ }^{11} \mathrm{~N}$-acetylaspartic acid accumulation leads to myelin vacuolization and astrocyte swelling, but neuronal cytoarchitecture is relatively well preserved. ${ }^{10,11}$ 
Table 1 Metabolic megalencephalies and their genetic causes ${ }^{\text {a }}$

\begin{tabular}{|l|l|l|}
\hline \multirow{5}{*}{ Organic acid disorders } & Glutaric aciduria, type I & GCDH \\
\cline { 2 - 3 } & L-2-Hydroxyglutaric aciduria & L2HGDH \\
\cline { 2 - 3 } & D-2-Hydroxyglutaric aciduria & D2HGDH \\
\hline \multirow{5}{*}{ Lysosomal storage diseases } & Hunter syndrome (mucopolysaccharidosis type II) & IDS \\
\cline { 2 - 3 } & Hurler syndrome (mucopolysaccharidosis type IH) & IDUA \\
\cline { 2 - 3 } & Sanfilippo syndrome (mucopolysaccharidosis III) & $\begin{array}{l}\text { SGSH (IIIA), } \\
\text { HAGLU (IIIB), } \\
\text { HGSNAT (IIIC), GNS (IIID) }\end{array}$ \\
\cline { 2 - 3 } & Maroteaux-Lamy syndrome (mucopolysaccharidosis type VI) & ARSB \\
\cline { 2 - 3 } & Sly syndrome (mucopolysaccharidosis VII) & GUSB \\
\cline { 2 - 3 } & Tay-Sachs disease & HEXA \\
\cline { 2 - 3 } & Sandhoff disease & HEXB \\
\cline { 2 - 3 } & Krabbe disease & GALC \\
\hline \multirow{5}{*}{ Leukoencephalopathies } & Canavan disease (N-acetylaspartic aciduria) & ASPA \\
\cline { 2 - 3 } & Alexander disease & GFAP \\
\cline { 2 - 3 } & $\begin{array}{l}\text { Megalencephalic leukoencephalopathy } \\
\text { with subcortical cysts (MLC) }\end{array}$ & MLC1, HEPACAM \\
\cline { 2 - 3 } & Leukoencephalopathy with vanishing white matter & EIF2B1, EIF2B2, EIF2B3, \\
\hline
\end{tabular}

Source: Adapted from Mirzaa GM, Poduri A. Megalencephaly and hemimegalencephaly: breakthroughs in molecular etiology. Am J Med Genet C Semin Med Genet 2014;166C(2):156-172.

ancluded are only syndromes associated with megalencephaly, and omitted are syndromes that might be associated with macrocephaly without brain enlargement.

Alexander disease is another disorder that preferentially affects glial cells. It is caused by mutations in the gene GFAP; this encodes glial fibrillary acidic protein, a structural component of astrocytes. ${ }^{8}$ Heterozygous gain-of-function mutations in GFAP cause accumulation of the GFAP protein within astrocytes into Rosenthal fibers, which are thought to be toxic to astrocytes and to cause cell death. ${ }^{12}$ There is also abnormal myelination, possibly secondary to disrupted astrocyte-oligodendrocyte interaction. ${ }^{13}$

In contrast to the leukodystrophies, there are also metabolic disorders that primarily affect neurons and lead to megalencephaly. For example, Tay-Sachs disease is a classic metabolic disorder caused by a loss of function of the enzyme $\beta$-hexosaminidase. There are two forms of $\beta$-hexosaminidase: $\beta$-hexosaminidase $A$ is a heterodimer comprised of an $\alpha$ and $\beta$ subunits and $\beta$-hexosaminidase $B$ is a homodimer comprised of two $\beta$ subunits. The HEXA gene encodes the $\alpha$ subunit of the $\beta$-hexosaminidase A enzyme, which converts GM2-ganglioside to GM3-ganglioside. Patients with Tay-Sachs disease have loss-of-function alleles for both copies of the HEXA gene, which eliminates the activity of $\beta$-hexosaminidase $A$ and leads to GM2 accumulation. These patients often present in infancy with hypotonia and failure to thrive, and progress to intractable epilepsy, spasticity, and death. GM2 specifically accumulates in neurons and forms of storage bodies that can be seen on electron microscopy. ${ }^{14}$ This metabolic defect results in progressive neuronal cell death and cerebral atrophy, although whether this is secondary to direct toxicity, secondary effects on neuronal development, or resultant inflammation is unclear. ${ }^{15}$ Interestingly, the residual activity of the enzyme is correlated with age of onset and severity of the phenotype in Tay-Sachs disease. ${ }^{14}$

Glutaric aciduria type 1 is caused by loss of function of the glutaryl-CoA dehydrogenase enzyme, encoded by the GCDH gene; it is associated with macrocephaly present in the neonatal period (- Fig. 1). Infants often have subtle initial symptoms of hypotonia, poor feeding, and irritability, but untreated patients will develop encephalopathic crises caused by a catabolic state in the setting of an infection. ${ }^{16}$ These crises often lead to basal ganglia damage, resulting in an irreversible dystonic-dyskinetic movement disorder. ${ }^{17}$ $\mathrm{GCDH}$ is a mitochondrial matrix protein that is expressed in neurons and involved in the catabolism of tryptophan, lysine, and hydroxylysine. Lack of GCDH activity results mainly in accumulation of glutaric acid and 3-hydroxyglutaric acid, and these metabolites have been implicated in alterations in energy metabolism and oxidative stress, which is thought to explain the neuronal vulnerability. ${ }^{18}$

Despite the seemingly simple paradigm of the metabolic megalencephalies, these disorders encompass a relatively heterogeneous group of disorders (-Table 1). Many of the disorders do involve a loss-of-function mutation in an enzyme, but this is not the rule, as evidenced by Alexander disease. In addition, these disorders affect general biochemical pathways but cause initial dysfunction in a single cell type. The mechanism of brain enlargement observed is not well understood and ranges from cell autonomous mechanisms involving enlargement of certain cells to inflammatory 
reactions to the presence of abnormal metabolites. Therefore, metabolic disorders represent several different pathophysiological mechanisms that contribute to brain enlargement and general dysfunction.

\section{Developmental Megalencephaly}

Developmental megalencephaly has also been referred to as anatomic megalencephaly or non-metabolic megalencephaly. Recent identification of mutations in genes regulating cell growth, migration, and replication has led to the recognition that developmental processes underlie these megalencephalies. ${ }^{3,4}$ Developmental megalencephalies are largely characterized by single gene mutations, with the most common mutations identified affecting the MTOR, Ras/MAPK, or SHH pathways (-Table 2). Asymmetric and focal lesions are more common in developmental megalencephalies, with the spectrum of abnormalities ranging from megalencephaly and hemimegalencephaly to the discrete tubers of tuberous sclerosis complex (TSC) (-Fig. 1). It is hypothesized that the variability in presentation may be in part due to somatic mosaicism. 6,7

\section{Somatic Mutation}

As opposed to the recessive mutations that commonly give rise to the metabolic megalencephalic disorders, de novo mutations have proven to be an important contributor to the developmental megalencephalies. ${ }^{3,6}$ As opposed to inherited mutations, de novo mutations are not found in both parent and offspring by standard genetic testing of DNA from leukocytes. A de novo mutation that arises after zygote formation is referred to as a somatic mutation, and will lead to mosaicism, a state in which various cells in an individual that can have different genotypes. These genetic alterations can happen at any point between gamete formation in the parent and organ development in the fetus, and can affect multiple organs or a single part of one organ, depending on the developmental stage at which they occur and the range of expression of the particular gene.

Somatic mutation in the brain has been hypothesized as one of many mechanisms to increase neuronal diversity, but studies have demonstrated a wide range of rates of somatic mutation in the brain. Multiple studies have used fluorescent in situ hybridization (FISH) to examine aneuploidy in the developing and mature brain and found rates of aneuploidy ranging between 10 to $35 \%{ }^{19}$ However, using single cell sorting and whole genome sequencing, aneuploidy could not be detected in control brains, whereas it was reliably detected in an individual with known trisomy $18 .{ }^{20}$ Efforts to determine the somatic mutation rate have led to relatively high estimates. ${ }^{21}$ However, there are disorders such as neurofibromatosis type 1 (NF1), in which somatic mutations in the skin are known to cause neurofibromas, and the burden of neurofibromas is much lower than what would be expected based on somatic mutation estimates alone, suggesting that there is additional complexity. Studies that have identified somatic mutations associated with brain malformations have obtained DNA directly from resected brain tissue from epilepsy surgery or found disease-causing mutations in peripheral leukocytes, inferring their presence in the brain. 22,23

\section{The mTOR Pathway}

The mTOR protein is a kinase that functions within a protein complex to integrate cell signaling and coordinate cell growth and differentiation. There are two functionally independent protein complexes that utilize mTOR, mTORC1 involved in cellular metabolism and growth and mTORC2 involved in cytoskeletal organization. ${ }^{24}$ Early studies in cortical tubers in TSC and some focal cortical dysplasias demonstrated that large ovoid cells with limited processes (giant cells or balloon cells) displayed selective activation of the mTOR pathway through increased amounts of phosphorylated ribosomal S6 kinase. ${ }^{25,26}$ Interestingly, some focal cortical dysplasias, cases of hemimegalencephaly, and TSC also demonstrate the presence of dysmorphic neurons, which have large soma and disorganized dendritic processes. ${ }^{27}$ These disorders also demonstrate varying degrees of disruption of cortical laminar organization. The similarities between these disorders both molecularly and pathologically suggest shared mTOR dysregulation. A recent study identified a somatic mutation in MTOR in a pathologically typical case of hemimegalencephaly in which there was increased phosphorylation of ribosomal S6 kinase in this case, consistent with an increase in mTOR activity. ${ }^{28}$ These data provide further evidence for the theory that dysregulation of MTOR is central to multiple forms of developmental megalencephaly.

There are many upstream molecules that regulate mTOR signaling, and many of these have been implicated in hemimegalencephaly (-Fig. 2). One key regulator of mTOR is the protein kinase AKT, which leads to activation of mTOR. Activating somatic mutations in AKT1 cause Proteus syndrome, characterized by somatic overgrowth of connective tissue and associated with hemimegalencephaly. ${ }^{29}$ AKT2 is associated with hypoinsulinemic hypoglycemia with hemihypertrophy, but has not been associated with megalencephaly at this time. ${ }^{30}$ AKT3 is highly expressed in the developing brain, and one study identified an activating mutation in AKT3 from dysplastic cortex of a patient with hemimegalencephaly, but not lymphocytes from the patient, demonstrating the presence of mosiacism. ${ }^{22}$ AKT3 mutations have also been shown to cause megalencephaly-polymicrogyria-polydactyly-hydrocephalus (MPPH) syndrome. ${ }^{31}$ Interestingly, deletions that presumably lead to loss of function of AKT3 are associated with microcephaly, ${ }^{32}$ demonstrating its key role in regulating proliferation and growth within the developing brain. Because there has been no target specificity associated with the AKT isoforms, the phenotypic variability seen within these disorders is likely due to a combination of the regulation of the gene leading to differential expression between tissues at different developmental time points and the exact population of cells affected by the mutation.

AKT is activated by recruitment to the cell membrane and binding to phosphatidylinositol-3,4,5-triphosphate (PIP3), which allows phosphorylation and subsequent activation of 
Table 2 Developmental megalencephalies and their genetic causes ${ }^{\text {a }}$

\begin{tabular}{|c|c|}
\hline \multicolumn{2}{|l|}{ PI3K-AKT-MTOR pathway } \\
\hline \multirow[t]{4}{*}{ PTEN $^{*}$} & Macrocephaly/autism syndrome \\
\hline & Bannayan-Riley-Ruvalcaba syndrome (BRRS) \\
\hline & Cowden syndrome \\
\hline & Lhermitte-Duclos syndrome \\
\hline \multirow[t]{3}{*}{ PI3KCA } & MCAP syndrome (megalencephaly-capillary malformation-polymicrogyria) \\
\hline & Klippel-Weber-Trenaunay syndrome \\
\hline & CLOVES (congenital lipomatous overgrowth, vascular malformations, and epidermal nevi) \\
\hline AKT3*, CCND2, PIK3R2 & MPPH syndrome (megalencephaly-polymicrogyria-polydactyly-hydrocephalus) \\
\hline \multirow[t]{2}{*}{$A K T 1^{*}$} & Proteus syndrome \\
\hline & Cowden syndrome \\
\hline STRADA & Pretzel syndrome or PMSE (polyhydramnios, megalencephaly, and symptomatic epilepsy) \\
\hline TSC $1^{*}$, TSC2 $^{*}$ & Tuberous sclerosis complex \\
\hline TBC1D7 & Macrocephaly/megalencephaly syndrome, autosomal recessive \\
\hline$M_{T O R^{*}}$ & Hemimegalencephaly \\
\hline \multicolumn{2}{|c|}{ Ras/mitogen-activated protein kinase (MAPK) pathway } \\
\hline NF1 & Neurofibromatosis 1 \\
\hline SPRED1 & Legius syndrome \\
\hline HRAS & Costello syndrome \\
\hline MAP2K2 & Cardiofaciocutaneous (CFC) syndrome \\
\hline NRAS, SOS1, RIT1, SHOC2 & Noonan syndrome \\
\hline KRAS, MAP2K1 & CFC syndrome, NS \\
\hline PTPN11 & LEOPARD syndrome, NS \\
\hline BRAF & LEOPARD syndrome, CFC syndrome, NS \\
\hline RAF1 & LEOPARD syndrome, NS \\
\hline RIN2 & MACS syndrome (macrocephaly, alopecia, cutis laxa, and scoliosis) \\
\hline \multicolumn{2}{|l|}{ SHH pathway } \\
\hline PTCH1 & Nevoid basal cell carcinoma (Gorlin) syndrome, 9q22.3 microdeletion \\
\hline KIF7 & Acrocallosal syndrome \\
\hline GLI3 & Greig cephalopolysyndactyly syndrome \\
\hline \multicolumn{2}{|l|}{ Transcriptional regulators } \\
\hline NSD1 & Soto syndrome \\
\hline$E Z H 2$ & Weaver syndrome \\
\hline \multirow[t]{2}{*}{ MED12 } & Opitz-Kaveggia (FG) syndrome \\
\hline & Lujan (Lujan-Fryns) syndrome \\
\hline \multicolumn{2}{|c|}{ Signaling molecules and mitotic regulators } \\
\hline GPC3 & Simpson-Golabi-Behmel syndrome \\
\hline OFD1 & Simpson-Golabi-Behmel syndrome (type 2) \\
\hline DIS3L2 & Perlman syndrome \\
\hline
\end{tabular}

Source: Adapted from Mirzaa GM, Poduri A. Megalencephaly and hemimegalencephaly: breakthroughs in molecular etiology. Am J Med Genet C Semin Med Genet 2014;166C(2):156-172.

${ }^{\mathrm{a}}$ The asterisks denote identified genes known to cause hemimegalencephaly to date.

the kinase. PIP3 is formed by phosphorylation of phosphatidylinositol-4,5-bisphosphate (PIP2) by PI3K, and this reaction is reversed by the phosphatase PTEN. PI3K is a heterodimer comprised of a catalytic and regulatory subunit, each of which have multiple isoforms encoded in the genome. PI3KCA encodes a catalytic subunit of the PI3K complex, and activating somatic mutation has been shown to cause CLOVES syndrome (congenital lipomatous overgrowth, vascular 


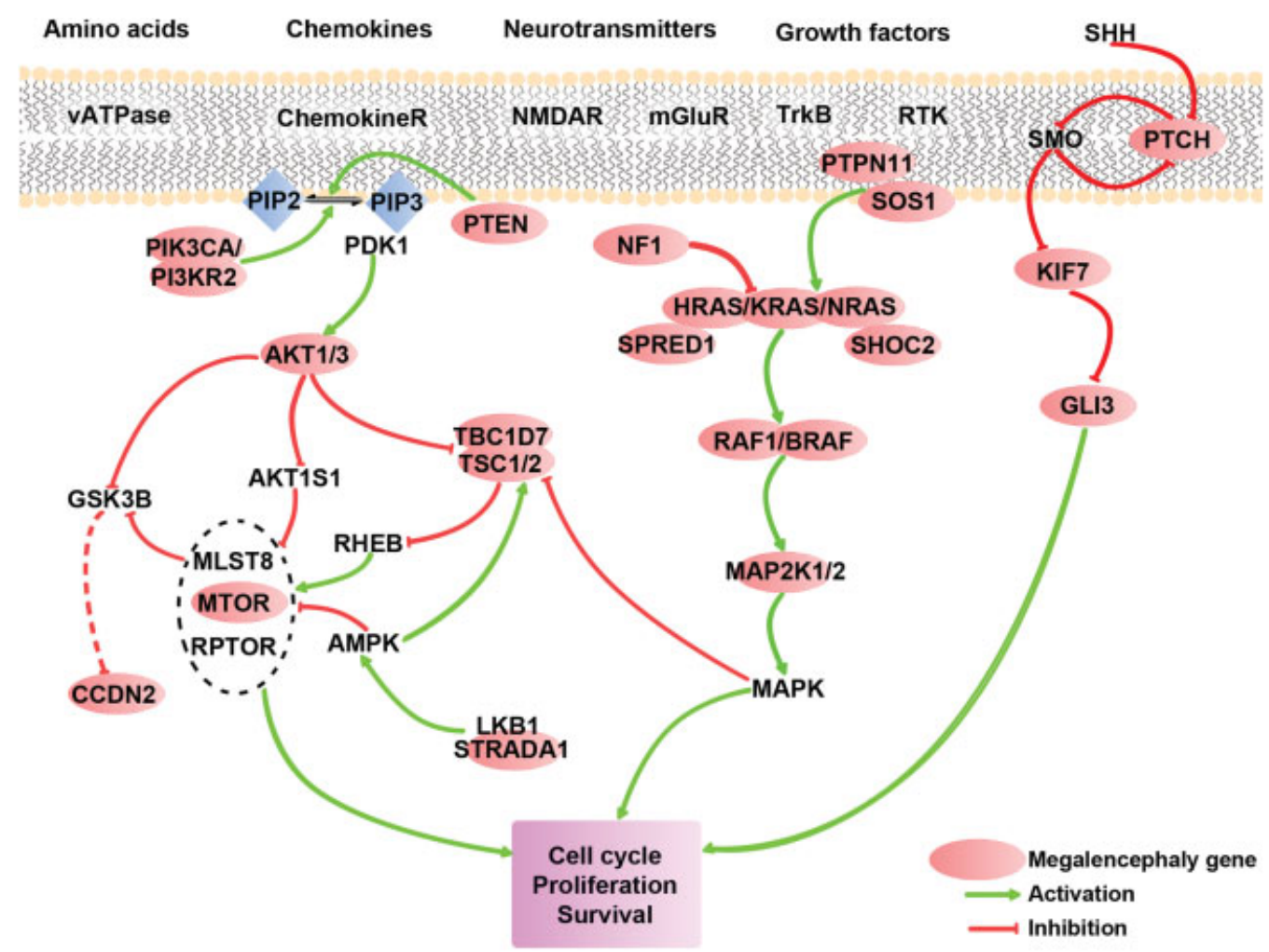

Fig. 2 Molecular signaling pathways whose dysregulation leads to developmental megalencephaly. The schematic shows key molecules in the mTOR, MAPK, and SHH signaling pathways. Categories of the extracellular signaling molecules are highlighted across the top with their receptors shown below. Specific interactions between the receptor categories and signaling mediators are not shown given the multitude and complexity of interactions. Interactions between signaling mediators are divided into activating (green arrows) and inhibitory (red blunt arrows). Signaling molecules whose dysregulation cause megalencephaly are highlighted by red circles. Some of the main downstream cellular effects are listed at the bottom.

malformations, epidermal nevus, spinal/skeletal anomalies/ scoliosis syndrome), characterized by focal overgrowth in various parts of the body (-Table 2$)^{33,34}$ Within the brain, activating somatic mutation of PI3KCA can cause megalencephaly-capillary malformation-polymicrogyria syndrome (MCAP) ${ }^{31}$ This syndrome is characterized by megalencephaly with connective tissue and vascular involvement to a variable severity and extent ( - Fig. 1). ${ }^{35}$ In addition, somatic mutation of PI3KCA has been shown to be associated with isolated hemimegalencephaly with polymicrogyria. ${ }^{28}$ One regulatory subunit isoform of the PI3K complex is encoded by PI3KR2, and mutations in this gene have been shown to be a common etiology for MPPH syndrome. ${ }^{31,36}$

Germline mutations of PTEN have been shown to cause Cowden syndrome and Bannayan-Riley-Ruvalcaba syndrome. Both syndromes are associated with overgrowth of connective tissue and multiple hamartomas with a susceptibility to multiple cancers. ${ }^{37,38}$ Affected patients may demonstrate bilateral megalencephaly with preservation of cytoarchitecture, but more focal involvement has been described as well. ${ }^{39}$ PTEN mutation is also one of the most common single gene mutations found in patients with autism and macrocephaly..$^{40,41}$

The genes TSC1 and TSC2 form a complex that regulates mTOR through inhibition of the mTOR-activator RHEB. Increased phosphorylated S6 kinase, a target of MTOR, has been shown as evidence of increased mTOR activity in cortical tubers. ${ }^{25}$ Tuberous sclerosis complex is an autosomal dominant disorder in which an individual carries only one functional copy of TSC1 or TSC2. These patients often have discrete cortical malformations or cortical tubers and epilepsy, as well as multiple skin findings, including hypopigmented macules and angiofibromas. ${ }^{42}$ In addition, they are predisposed to cancerous growths such as subependymal giant cell astrocytomas (SEGAs) and renal angiomyolipomas. Although these patients carry one defective copy of TSC1 or TSC2 in all of their cells, it has been shown that SEGAs and angiomyolipomas arise through a second mutation leading to a cell population that is functionally null for TSC1 or TSC2. ${ }^{43}$ Given the discrete, dysplastic nature of the cortical tubers in the brain, it has been hypothesized that these malformations also arise from a second mutation in TSC1 or TSC2. One study identified rare second hit mutations by sequencing bulk tissue from tubers. ${ }^{44}$ However, another study isolated and sequenced cells with high levels of phosphorylated S6-kinase and found second hit mutations in five of six cortical tubers studied, further suggesting that some tubers result from a cell population functionally null for one of the TSC genes. ${ }^{45}$ Interestingly, approximately $80 \%$ of TSC patients demonstrate cortical tubers on magnetic resonance imaging (MRI), ${ }^{46}$ and the requirement of a second hit would provide one explanation for this phenotypic variability. Another member of the TS complex is TBC1D7, and loss of this subunit leads to decreased association between TSC1 and TSC2. ${ }^{47}$ Loss of function 
mutations of this subunit have been described in patients with megalencephaly. ${ }^{48}$ Another mechanism of regulation of the TS complex is through activity of the kinase AMPK, which is regulated through a complex containing STK11, STRADA, and CAB39. Loss of function mutations in STRADA have been shown to cause the syndrome polyhydramnios, megalencephaly, and symptomatic epilepsy (PMSE), also known as Pretzel syndrome. ${ }^{49}$

mTOR is an important hub with multiple downstream effectors, regulating neuronal growth and development. However, mutations in the mTOR target CCND2 can cause $\mathrm{MPPH},{ }^{50}$ implicating the regulation of CCND2 by mTOR as critical to the development of megalencephaly. CCND2 is a cyclin that regulates exit from the G1 phase of the cell cycle, and one key mechanism of regulation of CCND2 is phosphorylation by GSK3 $\beta$ that leads to ubiquitination and degradation through the proteasome. ${ }^{51}$ However, mutations that cause MPPH abrogate this interaction, leading to accumulation of CCND2 and neuronal overgrowth. ${ }^{50}$ Interestingly, GSK3 $\beta$ is inhibited by mTOR and AKT, ${ }^{52,53}$ suggesting that dysregulation in the AKT/mTOR pathway could lead to CCND2 accumulation (-Fig. 2). Although each upstream component is part of a complex cellular signaling network, the developmental megalencephalies associated with alterations in mTOR signaling might share accumulation of CCND2, which would explain their pathological similarities.

Mutations in genes in the mTOR pathway that lead to increased mTOR signaling lead to disrupted cell growth and migration in the brain and other tissues. Although these disorders appear to be heterogeneous, the overgrowth abnormalities observed depend on the normal timing and location of gene expression. The normal expression of the gene determines the tissues that are vulnerable to mutation of the specific gene, illustrated by the differing phenotypes of AKT mutations. The tissues affected by the mutation are also critically dependent on the timing of the mutation in development, where earlier mutations in development will affect more tissues than later mutations.

\section{The Ras/MAPK Pathway}

The Ras/MAPK pathway transduces extracellular signals and regulates cell cycle, development, and senescence. There are germline mutations for multiple members of this signaling pathway that lead to multiple phenotypically overlapping syndromes, which demonstrate absolute or relative macrocephaly. These disorders are often associated with severe failure to thrive, and relative macrocephaly describes the situation in which a child's head circumference grows at an appropriate rate while length fails to keep up with the growth curve and crosses centiles. Imaging studies have also shown brain growth abnormalities in syndromes associated with increased Ras/MAPK signaling. ${ }^{54}$

The Ras family of genes encodes GTPases that interact with cellular receptors and signal through the intermediary proteins Raf and MAP2K/MEK leading to activation of MAPK/ERK (-Fig. 2). MAPK/MEK is a kinase that has multiple downstream targets that influence cellular functions through transcriptional and nontranscriptional mechanisms. Activat- ing mutations in Ras/MAPK pathway members have been shown to cause multiple overlapping syndromes, including Noonan syndrome, Noonan syndrome with multiple lentigines, Costello syndrome, cardiofaciocutaneous syndrome, NF1, and Legius syndrome. ${ }^{55}$ Noonan syndrome can be caused by heterozygous activating mutations in PTPN11, SOS1, NRAS, KRAS, RAF1, BRAF, SHOC2, or RIT1, ${ }^{56,57}$ but PTPN11 mutations are to date the most prevalent in this syndrome. ${ }^{58}$ PTPN11 and SOS1 lead to activation of the Ras proteins, whereas SHOC2 and RIT1 modify Raf signaling. Costello syndrome is caused by HRAS mutations, and cardiofaciocutaneous syndrome is caused by KRAS, BRAF, MAP2K1, and MAP2K2 mutations. ${ }^{55}$ However, patients affected with Noonan, Costello, and cardiofaciocutaneous syndromes share many common features besides brain abnormalities, including failure to thrive, developmental delay, craniofacial dysmorphologies, cardiac defects, and skin abnormalities. Developmental delay in cardiofaciocutaneous syndrome is more prevalent and severe than in Noonan syndrome, and Costello syndrome has characteristic skin findings, including excessive wrinkling and redundancy over the dorsum of the hands and feet. ${ }^{55}$ Noonan syndrome with multiple lentigines is phenotypically similar to Noonan syndrome, but there are abnormal genitalia and multiple lentigines. Interestingly, this syndrome is also caused by PTPN11 mutations, but these mutations occur in the phosphotyrosine phosphatase domain. ${ }^{59}$ NF1 is characterized by skin and eye findings, including cafe-au-lait macules, intertriginous freckling, neurofibromas, hamartomas of the iris (Lisch nodules), and optic pathway gliomas. Affected patients less commonly have developmental delays and craniofacial features similar to Noonan syndrome. This disorder is caused by germline gain of function mutations in the NF1 gene, and the NF1 protein (neurofibromin) inhibits Ras signaling. ${ }^{60}$ Legius syndrome is similar to NF1, but patients do not have neurofibromas or Lisch nodules and there is no increased risk of optic pathway gliomas. Heterozygous inactivating mutations in SPRED1 lead to Legius syndrome by altering Raf signaling. ${ }^{61}$

Ras/MAPK signaling in the developing telencephalon has been shown to promote the generation of neurons. ${ }^{62}$ In vivo and in vitro studies have demonstrated that reduction of PTPN11 decreases the number of neurons generated during development. ${ }^{63,64}$ However, a murine model of Noonan syndrome carrying a heterozygous activating mutation of PTPN11 showed a slight increase in neurons, but a substantial reduction in glial cells. ${ }^{65}$ It is difficult to reconcile these findings with the human syndrome, in which a substantial proportion of patients demonstrate megalencephaly. Another potential mechanism underlying the development of megalencephaly in these patients is an interaction with the mTOR pathway. ERK directly phosphorylates and inhibits TSC2 in tumor cell lines, leading to mTOR activation. ${ }^{66}$ Therefore, overactivation of Ras/MAPK signaling could allow unchecked mTOR signaling, leading to atypical cellular and cortical growth. However, patients with these Ras/MAPK signaling disorders rarely present with focal cortical dysplasia as seen in cases with mTOR signaling defects. ${ }^{67}$ In addition, an animal model of Noonan syndrome with a gain of function mutation 
of PTPN11 did not have increased phosphorylated S6, arguing against contributions of increased mTOR signaling in the model. ${ }^{65}$

Gliogenesis is also affected by aberrant Ras/MAPK signaling. Conditional transgenic animals carrying a gain of function mutation in PTPN11 in Olig2 + cells demonstrated increased oligodendroglial progenitors, ${ }^{65}$ seemingly contradictory to prior studies demonstrating reductions in glial cells. $^{64}$ The Olig2 driver used in the conditional transgenic animals preferentially targets ventral telencephalon, whereas the prior study had used in utero electroporation, suggesting regional or temporal differences in Ras/MAPK signaling leading to the observed differences. Animal models of NF1 inactivation or MAP2K1/MAP2K2 gain of function also show expansion of glial and glioprogenitor populations, ${ }^{68-70}$ reinforcing the role of Ras/MAPK signaling in gliogenesis. Patients with NF1 also have increased white matter volume with an abnormally thick corpus callosum. ${ }^{71}$ Therefore, alterations in glial progenitor proliferation and development may underlie some of the brain abnormalities seen in the Ras/ MAPK signaling disorders.

\section{Other Pathways and Indirect Regulators}

The Sonic Hedgehog pathway is involved in early pattern formation in the developing nervous system. The Sonic Hedgehog ligand (SHH) binds to its receptor PTCH1 removing the inhibition of PTCH1 on SMO, and SMO then is able to activate the GLI transcription factors (GLI1, GLI2, and GLI3) (-Fig. 2). Grieg cephalopolysyndactyly syndrome (GCPS) can be caused by mutations in GLI3 and is characterized by macrocephaly, craniofacial dysmorphology, and limb malformations. ${ }^{72}$ Mutations identified thus far have led to loss of one functional allele; therefore, haploinsufficiency of this transcription factor is thought to cause this syndrome. ${ }^{72}$ However, animal models with loss of function of GLI3 have severely reduced telencephalon, ${ }^{73}$ whereas animals with a GLI3 hypermorphic allele have larger forebrains. ${ }^{74}$ Interestingly, the increased forebrain size was thought to be mediated by a shorter cell cycle in progenitors caused by upregulation of the GLI3 target CCND1. ${ }^{74}$ Mutations in the SHH receptor PTCH1 leading to haploinsufficiency can cause nevoid basal cell carcinoma syndrome. This syndrome is characterized by multiple basal cell carcinomas, as well as skeletal and facial features. ${ }^{75}$ These disorders highlight the importance of dysregulation of the SHH signaling pathway in contributing to brain growth abnormalities.

There are many other genes associated with developmental megalencephaly; many of them can affect either or both mTOR or Ras/MAPK signaling. Sotos and Weaver syndromes have characteristic craniofacial dysmorphologies, including high broad forehead and prominent chin, overgrowth with tallness being more common than macrocephaly, and intellectual disability (- Fig. 1) $)^{76}$ Sotos and Weaver syndromes are associated with NSD1 and EZH2 mutations, respectively; both genes encode histone methyltransferases involved in epigenetic regulation. Sotos syndrome is associated with haploinsufficiency of NSD1, and Weaver syndrome is associated with missense mutations in EZH2, suggesting loss of function of these genes as the pathological mechanism underlying these disorders. NSD1 and EZH2 have been suggested to activate both MTOR and MAPK signaling through modulating expression of upstream molecules. For example, EZH2 has been shown to suppress PTEN and lead to increased proliferation in neuroprogenitors. ${ }^{77}$ EZH2 and NSD1 have been shown to activate MAPK signaling through CXXC4 and RASIP1, respectively. ${ }^{78,79}$ Therefore, loss of function of these genes likely has complex effects on the mTOR and Ras/MAPK pathways, leading to brain growth abnormalities.

Although there are many genes with varying functions associated with megalencephaly, many appear to modify mTOR and/or MAPK signaling pathways. This suggests that these genes may cause brain enlargement by converging onto known mechanisms. However, this is not surprising given the central role of mTOR and MAPK in cell-signaling networks. The phenotypic variability seen between these disorders is likely due to either variable penetrance or the degree that specific pathways are modulated by these mutations.

\section{Treatment}

Most of the metabolic and developmental megalencephalies are associated with developmental delay and epilepsy, as well as other disease-specific manifestations. Developmental delay and regression in certain cases may be due to neuronal cell death, but in some disorders, they appear to be related to medically intractable epilepsy. In some cases with focal abnormalities of cerebral development, epilepsy surgery can be an effective treatment. ${ }^{80}$ In addition, epilepsy surgery in TSC can help in terms of both seizure freedom and developmental improvements. ${ }^{81}$ However, for patients who are not surgical candidates, the mainstay of treatment is antiepileptic medication.

Given the important role of mTOR in the development of several different forms of megalencephaly, there has been increasing interest in using mTOR inhibitors as medical therapy. Some studies in animal models suggest that mTOR inhibition with rapamycin may help to prevent epileptogenesis, although this model was not related to the disorders of mTOR signaling. ${ }^{82}$ In a small study of patients with TSC, another mTOR inhibitor, everolimus, was suggested to be beneficial as an antiepileptic medication for some patients. ${ }^{83,84}$ However, more study is required to determine whether this effect is reproducible. Ultimately, there may be a role for specific mTOR inhibitors applicable to a broader range of developmental epilepsy associated with megalencephaly.

\section{Conclusions}

Syndromes associated with brain growth abnormalities comprise a diverse set of disorders. We propose refining the characterization of these disorders by dividing them into metabolic and developmental causes of megalencephaly. Metabolic megalencephalies have been well characterized, and these disorders mostly involve defects in cellular metabolic pathways, leading to accumulation of abnormal metabolites. There is a diverse range of pathways and 
pathophysiological mechanisms that contribute to these disorders, but most result in progressive cellular dysfunction and death. Developmental megalencephalies are caused by defects in signaling pathways that alter neuronal replication, growth, or migration leading to abnormal development of the brain. The mTOR, Ras/MAPK, and SHH pathways represent the most commonly affected pathways (-Fig. 2), and mutations in some of these molecules at any point between gamete formation and organogenesis can lead to disease. Postzygotic mutations that can affect only part of the brain explain the ability of a genetic disorder to cause asymmetric abnormalities. At this point, it is unclear whether the seizures, developmental disabilities, or behavioral symptoms observed are secondary to an ongoing molecular dysregulation or a product of an abnormally developed brain. However, the understanding of the molecular etiology and pathophysiological mechanisms of these disorders is providing new avenues for investigation and intervention.

\section{References}

1 DeMyer W. Megalencephaly: types, clinical syndromes, and management. Pediatr Neurol 1986;2(6):321-328

2 Williams CA, Dagli A, Battaglia A. Genetic disorders associated with macrocephaly. Am J Med Genet A 2008;146A(15):2023-2037

3 Mirzaa GM, Poduri A. Megalencephaly and hemimegalencephaly: breakthroughs in molecular etiology. Am J Med Genet C Semin Med Genet 2014;166C(2):156-172

4 Guerrini R, Dobyns WB. Malformations of cortical development: clinical features and genetic causes. Lancet Neurol 2014;13(7): 710-726

5 Barkovich AJ, Guerrini R, Kuzniecky RI, Jackson GD, Dobyns WB. A developmental and genetic classification for malformations of cortical development: update 2012. Brain 2012;135(Pt 5): 1348-1369

6 Poduri A, Evrony GD, Cai X, Walsh CA. Somatic mutation, genomic variation, and neurological disease. Science 2013;341(6141): 1237758

7 Baek ST, Gibbs EM, Gleeson JG, Mathern GW. Hemimegalencephaly, a paradigm for somatic postzygotic neurodevelopmental disorders. Curr Opin Neurol 2013;26(2):122-127

8 Rodriguez D. Leukodystrophies with astrocytic dysfunction. Handb Clin Neurol 2013;113:1619-1628

9 Traeger EC, Rapin I. The clinical course of Canavan disease. Pediatr Neurol 1998;18(3):207-212

10 Baslow MH. Canavan's spongiform leukodystrophy: a clinical anatomy of a genetic metabolic CNS disease. J Mol Neurosci 2000;15(2):61-69

11 Matalon R, Michals K, Sebesta D, Deanching M, Gashkoff P, Casanova J. Aspartoacylase deficiency and N-acetylaspartic aciduria in patients with Canavan disease. Am J Med Genet 1988;29(2): 463-471

12 Quinlan RA, Brenner M, Goldman JE, Messing A. GFAP and its role in Alexander disease. Exp Cell Res 2007;313(10):2077-2087

13 Mignot C, Boespflug-Tanguy O, Gelot A, Dautigny A, Pham-Dinh D, Rodriguez D. Alexander disease: putative mechanisms of an astrocytic encephalopathy. Cell Mol Life Sci 2004;61(3):369-385

14 Sandhoff K, Harzer K. Gangliosides and gangliosidoses: principles of molecular and metabolic pathogenesis. J Neurosci 2013;33(25): 10195-10208

15 Walkley SU. Neurobiology and cellular pathogenesis of glycolipid storage diseases. Philos Trans R Soc Lond B Biol Sci 2003; 358(1433):893-904
16 Hedlund GL, Longo N, Pasquali M. Glutaric acidemia type 1. Am J Med Genet C Semin Med Genet 2006;142C(2):86-94

17 Strauss KA, Puffenberger EG, Robinson DL, Morton DH. Type I glutaric aciduria, part 1: natural history of 77 patients. Am J Med Genet C Semin Med Genet 2003;121C(1):38-52

18 Jafari P, Braissant O, Bonafé L, Ballhausen D. The unsolved puzzle of neuropathogenesis in glutaric aciduria type I. Mol Genet Metab 2011;104(4):425-437

19 Bushman DM, Chun J. The genomically mosaic brain: aneuploidy and more in neural diversity and disease. Semin Cell Dev Biol 2013;24(4):357-369

20 Cai X, Evrony GD, Lehmann HS, et al. Single-cell, genome-wide sequencing identifies clonal somatic copy-number variation in the human brain. Cell Reports 2014;8(5):1280-1289

21 Lynch M. Evolution of the mutation rate. Trends Genet 2010;26(8): 345-352

22 Poduri A, Evrony GD, Cai X, et al. Somatic activation of AKT3 causes hemispheric developmental brain malformations. Neuron 2012; 74(1):41-48

23 Jamuar SS, Lam AT, Kircher M, et al. Somatic mutations in cerebral cortical malformations. N Engl J Med 2014;371(8):733-743

24 Lipton JO, Sahin M. The neurology of mTOR. Neuron 2014;84(2): 275-291

25 Crino PB. Molecular pathogenesis of tuber formation in tuberous sclerosis complex. J Child Neurol 2004;19(9):716-725

26 Baybis M, Yu J, Lee A, et al. mTOR cascade activation distinguishes tubers from focal cortical dysplasia. Ann Neurol 2004;56(4): 478-487

27 Lim KC, Crino PB. Focal malformations of cortical development: new vistas for molecular pathogenesis. Neuroscience 2013; 252:262-276

28 Lee JH, Huynh M, Silhavy JL, et al. De novo somatic mutations in components of the PI3K-AKT3-mTOR pathway cause hemimegalencephaly. Nat Genet 2012;44(8):941-945

29 Griffiths PD, Welch RJ, Gardner-Medwin D, Gholkar A, McAllister $\mathrm{V}$. The radiological features of hemimegalencephaly including three cases associated with Proteus syndrome. Neuropediatrics 1994;25(3):140-144

30 Arya VB, Flanagan SE, Schober E, Rami-Merhar B, Ellard S, Hussain K. Activating AKT2 mutation: hypoinsulinemic hypoketotic hypoglycemia. J Clin Endocrinol Metab 2014;99(2):391-394

31 Rivière JB, Mirzaa GM, O'Roak BJ, et al; Finding of Rare Disease Genes (FORGE) Canada Consortium. De novo germline and postzygotic mutations in AKT3, PIK3R2 and PIK3CA cause a spectrum of related megalencephaly syndromes. Nat Genet 2012;44(8): 934-940

32 Hill AD, Chang BS, Hill RS, et al. A 2-Mb critical region implicated in the microcephaly associated with terminal $1 \mathrm{q}$ deletion syndrome. Am J Med Genet A 2007;143A(15):1692-1698

33 Lindhurst MJ, Parker VE, Payne F, et al. Mosaic overgrowth with fibroadipose hyperplasia is caused by somatic activating mutations in PIK3CA. Nat Genet 2012;44(8):928-933

34 Kurek KC, Luks VL, Ayturk UM, et al. Somatic mosaic activating mutations in PIK3CA cause CLOVES syndrome. Am J Hum Genet 2012;90(6):1108-1115

35 Mirzaa GM, Paciorkowski AR, Smyser CD, Willing MC, Lind AC, Dobyns WB. The microcephaly-capillary malformation syndrome. Am J Med Genet A 2011;155A(9):2080-2087

36 Nakamura K, Kato M, Tohyama J, et al. AKT3 and PIK3R2 mutations in two patients with megalencephaly-related syndromes: MCAP and MPPH. Clin Genet 2014;85(4):396-398

37 Liaw D, Marsh DJ, Li J, et al. Germline mutations of the PTEN gene in Cowden disease, an inherited breast and thyroid cancer syndrome. Nat Genet 1997;16(1):64-67

38 Marsh DJ, Dahia PL, Zheng Z, et al. Germline mutations in PTEN are present in Bannayan-Zonana syndrome. Nat Genet 1997;16(4): 333-334 
39 Merks JH, de Vries LS, Zhou XP, et al. PTEN hamartoma tumour syndrome: variability of an entity. J Med Genet 2003;40(10):e111

40 Butler MG, Dasouki MJ, Zhou XP, et al. Subset of individuals with autism spectrum disorders and extreme macrocephaly associated with germline PTEN tumour suppressor gene mutations. J Med Genet 2005;42(4):318-321

41 Buxbaum JD, Cai G, Chaste P, et al. Mutation screening of the PTEN gene in patients with autism spectrum disorders and macrocephaly. Am J Med Genet B Neuropsychiatr Genet 2007;144B(4): 484-491

42 Crino PB, Nathanson KL, Henske EP. The tuberous sclerosis complex. N Engl J Med 2006;355(13):1345-1356

43 Henske EP, Wessner LL, Golden J, et al. Loss of tuberin in both subependymal giant cell astrocytomas and angiomyolipomas supports a two-hit model for the pathogenesis of tuberous sclerosis tumors. Am J Pathol 1997;151(6):1639-1647

44 Qin W, Chan JA, Vinters HV, et al. Analysis of TSC cortical tubers by deep sequencing of TSC1, TSC2 and KRAS demonstrates that smal second-hit mutations in these genes are rare events. Brain Pathol 2010;20(6):1096-1105

45 Crino PB, Aronica E, Baltuch G, Nathanson KL. Biallelic TSC gene inactivation in tuberous sclerosis complex. Neurology 2010; 74(21):1716-1723

46 Crino PB. Evolving neurobiology of tuberous sclerosis complex. Acta Neuropathol 2013;125(3):317-332

47 Dibble CC, Elis W, Menon S, et al. TBC1D7 is a third subunit of the TSC1-TSC2 complex upstream of mTORC1. Mol Cell 2012;47(4): 535-546

48 Capo-Chichi JM, Tcherkezian J, Hamdan FF, et al. Disruption of TBC1D7, a subunit of the TSC1-TSC2 protein complex, in intellectual disability and megalencephaly. J Med Genet 2013;50(11): 740-744

49 Puffenberger EG, Strauss KA, Ramsey KE, et al. Polyhydramnios, megalencephaly and symptomatic epilepsy caused by a homozygous 7-kilobase deletion in LYK5. Brain 2007;130(Pt 7): 1929-1941

50 Mirzaa GM, Parry DA, Fry AE, et al; FORGE Canada Consortium. De novo CCND2 mutations leading to stabilization of cyclin D2 cause megalencephaly-polymicrogyria-polydactyly-hydrocephalus syndrome. Nat Genet 2014;46(5):510-515

51 Kida A, Kakihana K, Kotani S, Kurosu T, Miura O. Glycogen synthase kinase-3beta and p38 phosphorylate cyclin D2 on Thr280 to trigger its ubiquitin/proteasome-dependent degradation in hematopoietic cells. Oncogene 2007;26(46): 6630-6640

52 Zhang HH, Lipovsky AI, Dibble CC, Sahin M, Manning BD. S6K1 regulates GSK3 under conditions of mTOR-dependent feedback inhibition of Akt. Mol Cell 2006;24(2):185-197

53 Cross DA, Alessi DR, Cohen P, Andjelkovich M, Hemmings BA. Inhibition of glycogen synthase kinase- 3 by insulin mediated by protein kinase B. Nature 1995;378(6559):785-789

54 Gripp KW, Hopkins E, Doyle D, Dobyns WB. High incidence of progressive postnatal cerebellar enlargement in Costello syndrome: brain overgrowth associated with HRAS mutations as the likely cause of structural brain and spinal cord abnormalities. Am J Med Genet A 2010;152A(5):1161-1168

55 Rauen KA. The RASopathies. Annu Rev Genomics Hum Genet 2013;14:355-369

56 Romano AA, Allanson JE, Dahlgren J, et al. Noonan syndrome: clinical features, diagnosis, and management guidelines. Pediatrics 2010;126(4):746-759

57 Aoki Y, Niihori T, Banjo T, et al. Gain-of-function mutations in RIT1 cause Noonan syndrome, a RAS/MAPK pathway syndrome. Am J Hum Genet 2013;93(1):173-180

58 Tartaglia M, Mehler EL, Goldberg R, et al. Mutations in PTPN11, encoding the protein tyrosine phosphatase SHP-2, cause Noonan syndrome. Nat Genet 2001;29(4):465-468
59 Digilio MC, Conti E, Sarkozy A, et al. Grouping of multiplelentigines/LEOPARD and Noonan syndromes on the PTPN11 gene. Am J Hum Genet 2002;71(2):389-394

60 Williams VC, Lucas J, Babcock MA, Gutmann DH, Korf B, Maria BL. Neurofibromatosis type 1 revisited. Pediatrics 2009;123(1): 124-133

61 Brems H, Chmara M, Sahbatou M, et al. Germline loss-of-function mutations in SPRED1 cause a neurofibromatosis 1-like phenotype. Nat Genet 2007;39(9):1120-1126

62 Ménard C, Hein P, Paquin A, et al. An essential role for a MEK-C/EBP pathway during growth factor-regulated cortical neurogenesis. Neuron 2002;36(4):597-610

63 Ke Y, Zhang EE, Hagihara K, et al. Deletion of Shp2 in the brain leads to defective proliferation and differentiation in neural stem cells and early postnatal lethality. Mol Cell Biol 2007;27(19): 6706-6717

64 Gauthier AS, Furstoss O, Araki T, et al. Control of CNS cell-fate decisions by SHP-2 and its dysregulation in Noonan syndrome. Neuron 2007;54(2):245-262

65 Ehrman LA, Nardini D, Ehrman S, et al. The protein tyrosine phosphatase Shp2 is required for the generation of oligodendrocyte progenitor cells and myelination in the mouse telencephalon. J Neurosci 2014;34(10):3767-3778

66 Ma L, Chen Z, Erdjument-Bromage H, Tempst P, Pandolfi PP. Phosphorylation and functional inactivation of TSC2 by Erk implications for tuberous sclerosis and cancer pathogenesis. Cell 2005;121(2):179-193

67 Saito Y, Sasaki M, Hanaoka S, Sugai K, Hashimoto T. A case of Noonan syndrome with cortical dysplasia. Pediatr Neurol 1997; 17(3):266-269

68 Bennett MR, Rizvi TA, Karyala S, McKinnon RD, Ratner N. Aberrant growth and differentiation of oligodendrocyte progenitors in neurofibromatosis type 1 mutants. J Neurosci 2003;23(18): 7207-7217

$69 \mathrm{Li}$ X, Newbern JM, Wu Y, et al. MEK is a key regulator of gliogenesis in the developing brain. Neuron 2012;75(6):1035-1050

70 Hegedus B, Dasgupta B, Shin JE, et al. Neurofibromatosis-1 regulates neuronal and glial cell differentiation from neuroglial progenitors in vivo by both cAMP- and Ras-dependent mechanisms. Cell Stem Cell 2007;1(4):443-457

71 Steen RG, Taylor JS, Langston JW, et al. Prospective evaluation of the brain in asymptomatic children with neurofibromatosis type 1: relationship of macrocephaly to $\mathrm{T} 1$ relaxation changes and structural brain abnormalities. AJNR Am J Neuroradiol 2001; 22(5):810-817

72 Jamsheer A, Sowińska A, Trzeciak T, Jamsheer-Bratkowska M, Geppert A, Latos-Bieleńska A. Expanded mutational spectrum of the GLI3 gene substantiates genotype-phenotype correlations. J Appl Genet 2012;53(4):415-422

73 Theil T, Alvarez-Bolado G, Walter A, Rüther U. Gli3 is required for Emx gene expression during dorsal telencephalon development. Development 1999;126(16):3561-3571

74 Wilson SL, Wilson JP, Wang C, Wang B, McConnell SK. Primary cilia and Gli3 activity regulate cerebral cortical size. Dev Neurobiol 2012;72(9):1196-1212

75 Fujii K, Miyashita T. Gorlin syndrome (nevoid basal cell carcinoma syndrome): update and literature review. Pediatr Int 2014;56(5): 667-674

76 Tatton-Brown K, Rahman N. The NSD1 and EZH2 overgrowth genes, similarities and differences. Am J Med Genet C Semin Med Genet 2013;163C(2):86-91

77 Zhang J, Ji F, Liu Y, et al. Ezh2 regulates adult hippocampal neurogenesis and memory. J Neurosci 2014;34(15): 5184-5199

78 Visser R, Landman EB, Goeman J, Wit JM, Karperien M. Sotos syndrome is associated with deregulation of the MAPK/ERKsignaling pathway. PLoS ONE 2012;7(11):e49229 
79 Lu H, Jin W, Sun J, et al. New tumor suppressor CXXC finger protein 4 inactivates mitogen activated protein kinase signaling. FEBS Lett 2014;588(18):3322-3326

80 Wyllie E, Comair YG, Kotagal P, Bulacio J, Bingaman W, Ruggieri P. Seizure outcome after epilepsy surgery in children and adolescents. Ann Neurol 1998;44(5):740-748

81 Shahid A. Resecting the epileptogenic tuber: what happens in the long term? Epilepsia 2013;54(Suppl 9): $135-138$
82 Raffo E, Coppola A, Ono T, Briggs SW, Galanopoulou AS. A pulse rapamycin therapy for infantile spasms and associated cognitive decline. Neurobiol Dis 2011;43(2):322-329

83 Wiegand G, May TW, Ostertag P, Boor R, Stephani U, Franz DN. Everolimus in tuberous sclerosis patients with intractable epilepsy: a treatment option? Eur J Paediatr Neurol 2013;17(6):631-638

84 Krueger DA, Wilfong AA, Holland-Bouley $\mathrm{K}$, et al. Everolimus treatment of refractory epilepsy in tuberous sclerosis complex. Ann Neurol 2013;74(5):679-687 